\title{
Novel Polyhedral Finite Elements for Numerical Analysis
}

\author{
Logah Perumal*, C. P. Tso, Lim Thong Leng \\ Faculty of Engineering and Technology, Multimedia University, Jalan Ayer Keroh Lama, Bukit Beruang, \\ 75450 Melaka, Malaysia. \\ * Corresponding author. Tel.: 06-2523287 email: logah.perumal@mmu.edu.my \\ Manuscript submitted July 10, 2017; accepted September 8, 2017. \\ doi: 10.17706/ijcee.2017.9.2.492-501
}

\begin{abstract}
Novel polyhedral elements termed as virtual node polyhedral element (VPHE) have been formulated based on virtual node method. The VPHEs are able to take arbitrary forms with arbitrary number of nodes and sides. Formulation of the VPHE is provided in this work, together with three examples that demonstrate utilization of the new element in carrying out numerical analyses. The first and second examples involve determination of steady-state temperature distribution (heat transfer phenomena) for two different geometries while the third example involves determination of Young's modulus for carbon nanotubes (solid mechanics). Three different meshes with increasing number of finite elements (VPHEs) are utilized in examples 1 and 2. Example 3 is simulated by representing each cell of a carbon nanotube with a VPHE element. This is done for two different configurations of carbon nanotube (Armchair and Zigzag). Simulation results show that the VPHEs are able to produce converging solutions towards the analytical or experimental solutions for all three cases considered in this work.
\end{abstract}

Key words: Finite element method, polyhedral finite element, virtual node method, numerical analysis.

\section{Introduction}

Finite element method (FEM) is a numerical method that is vastly utilized to solve problems which are difficult to be solved analytically. Conventional elements have fixed number of sides and faces. Thereafter, polygonal elements with arbitrary number of nodes and sides have been formulated (for 2-dimensional cases) and later extended to polyhedral elements for 3-dimensional cases. Many methods have been proposed to generate polyhedral elements [1]. Objectives of the current work are to develop and validate new polyhedral elements based on the virtual node method [2]. The new polyhedral elements are termed virtual node polyhedral element (VPHE). Three numerical examples are provided to show performance of the VPHEs in numerical analyses. Simulation results from analysis of steady state temperature distribution within a square plate show advantages of VPHEs in meshing surface of a curved geometry and higher number of nodes generated. The VPHEs are also shown to be more suitable in simulation of carbon nanotubes.

\section{Methods}

Element equations for a VPHE are formulated by coupling nodal shape functions of tetrahedral element with least-squares approximation. For that reason, the arbitrary VPHE is partitioned into $t$ number of tetrahedral elements. Each plane of the VPHE is partitioned into triangles which share a common center, $C$. Number of triangles on a face is equal to total number of nodes on the particular face. The fourth node for 
each triangle (vertex, $V$ ) which forms tetrahedral is located at the center of the VPHE. An example of partitioning of an arbitrary VPHE is shown in Fig. 1.
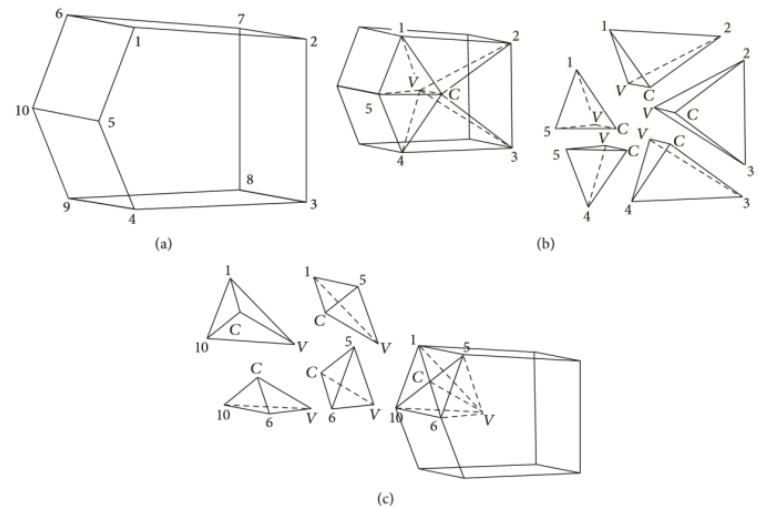

Fig. 1. Geometry of an arbitrary polyhedral element (VPHE): (a) a VPHE with 10 nodes and 7 faces (b) partitioning of the face 1-2-3-4-5 into 5 tetrahedral elements (c) partitioning of the face 1-5-6-10 into 4 tetrahedral elements.

Linear tetrahedral element is utilized to partition the VPHE, since this element has linear sides and the shape function for a node is always independent from the other nodes, for any geometry configuration. The vertex, $V$, acts as an imaginary or virtual node, which is utilized to combine all the tetrahedral elements to form the VPHE. The node $V$ is utilized only to develop shape functions for the VPHE nodes and does not contribute to computational cost. Field variable within each tetrahedral element within the polyhedron are calculated by using the formula:

$$
\varphi^{\left[T_{v}\right]}=\phi_{i}^{\left[T_{v}\right]} \varphi_{1}+\phi_{j}^{\left[T_{v}\right]} \varphi_{2}+\phi_{k}^{\left[T_{v}\right]} \varphi_{3}+\phi_{l}^{\left[T_{v}\right]} \varphi_{4}
$$

where $T_{v}$ represents a specific tetrahedron within the polyhedron (i.e., $T_{1}, T_{2}, T_{3}, \ldots, T_{v}, \ldots T_{t}$ ), $\phi_{i}^{\left[T_{v}\right]}, \phi_{j}^{\left[T_{v}\right]}, \phi_{k}^{\left[T_{v}\right]}$, and $\phi_{l}^{\left[T_{v}\right]}$ represent nodal shape functions of a specific tetrahedron for nodes $i, j, k$ and $l$, respectively, and $\varphi_{1}, \varphi_{2}, \varphi_{3}$, and $\varphi_{4}$ represent field variables for the specific tetrahedral nodes $i, j, k$ and $l$, respectively. Nodes $i, j$ and $k$ are located on the outer surface of the polyhedron, while node $l$ is located at inside of the polyhedron. Node $l$, which is located at inside of the polyhedron is the common point shared by all the tetrahedrons (which form the polyhedron). Location of the common point $\left(x_{l}, y_{l}, z_{l}\right)$ is simply the centre of the polyhedron (node $V$ in Fig. 1).

The VPHEs are first validated through patch tests. The patch consists of 6 VPHE elements with combination of 6, 7, and 8 faces as shown in Fig. 2. Material properties used in the patch test are Young's modulus of elasticity, $E=10^{6} \mathrm{~Pa}$; Poisson ratio, $v=0.3$; and thermal conductivities, $k_{x}=k_{y}=k_{z}=25 \mathrm{~W} / \mathrm{m}^{0} \mathrm{C}$.

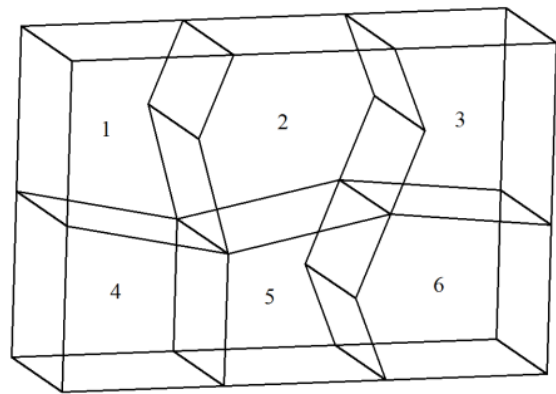

Fig. 2. Patch test for the VPHE elements. 
External nodes for each case are assigned with fixed displacement/temperature values according to the linear functions:

$$
\left.\begin{array}{ll}
u_{x, i}=X_{i} \\
\begin{array}{l}
v_{y, i} \\
w_{z, i}=Y_{i} \\
T_{i}=X_{i}+Y_{i}+Z_{i}
\end{array}
\end{array}\right\} \text { for solid mechanics }
$$

The linear displacements/temperatures on the external nodes represent constant strain/heat flux within the interior of the elements. The displacements/temperatures of the inner nodes are calculated using finite element equations and the difference between the calculated and exact values are found using $L^{2}$ error norm in displacement/temperature using (3):

$$
e=\sqrt{\frac{\int\left(\left(\varphi_{x, i}, \varphi_{y, i}, \varphi_{z, i}\right)^{\text {exact }}-\left(\varphi_{x, i}, \varphi_{y, i}, \varphi_{z, i}\right)^{\text {calculataed }}\right)^{2} d \Omega}{\int_{\Omega}\left(\left(\varphi_{x, i}, \varphi_{y, i}, \varphi_{z, i}\right)^{\text {exact }}\right)^{2} d \Omega}}
$$

The patch test results for the VPHE elements are shown in Table 1.

Table 1. Patch Test Results for VPHE Elements (L2 Error Norm in Displacement and Temperature)

\begin{tabular}{|l|l|l|}
\hline $\begin{array}{l}\text { Number of } \\
\text { elements in the } \\
\text { patch }\end{array}$ & $\begin{array}{l}\text { Error norm in } \\
\text { displacement }\end{array}$ & $\begin{array}{l}\text { Error norm in } \\
\text { temperature }\end{array}$ \\
\hline 6 & $3.031 \times 10^{-3}$ & $5.787 \times 10^{-4}$ \\
\hline
\end{tabular}

It is seen that the range for the error norms of the VPHE elements are similar to those obtained using Wachspress and mean value coordinate for 2-dimensions as reported in [3] and 3D PFEM as reported in [4] for 3-dimensions. It is also observed that the error norm in heat transfer phenomena is lower. This is caused by higher degree of freedom in solid mechanics.

\section{Numerical Applications}

Three numerical examples are selected to demonstrate performance of the VPHEs. The first example (Case 1) is the calculation of steady state temperature distribution for a hollow cylinder which is subjected to fixed temperature loads. Geometry of the problem domain is shown in Fig. 3 (a). Dimensions of the hollow cylinder are: inner radius $=1 \mathrm{~m}$, outer radius $=4 \mathrm{~m}$, and thickness of the cylinder $=1 \mathrm{~m}$. Due to the symmetry, only quarter of the hollow cylinder is analysed, as shown in Fig. 3 (b).

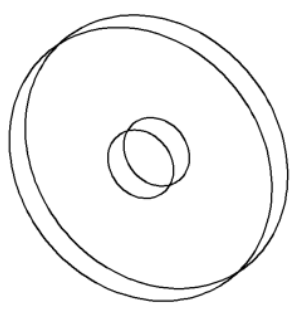

(a)

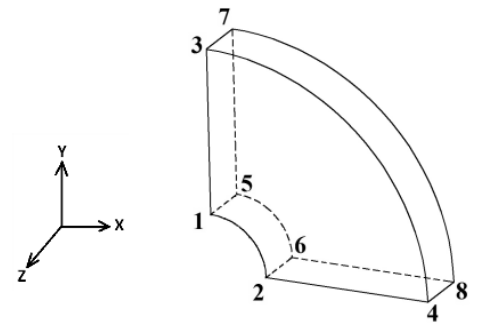

(b)

Fig. 3. Problem geometry for Case 1: (a) a hollow cylinder (b) quarter of the hollow cylinder.

The temperature of the inner surface, 1-2-5-6 is fixed at $500^{\circ} \mathrm{C}$ and the outer surface, 3-4-7-8 is fixed at $100^{\circ} \mathrm{C}$ (Fig. 3(b)). Other surfaces are considered adiabatic. Three different meshes (with increasing number 
of elements and global nodes) are considered: $2 \times 2 \times 1$ mesh, $3 \times 3 \times 1$ mesh and $4 \times 4 \times 1$ mesh as shown in Fig. 4 . Simulation results are shown in next section.

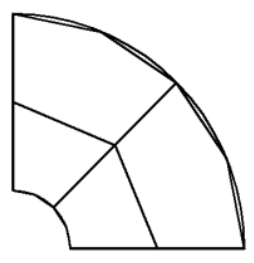

(a)

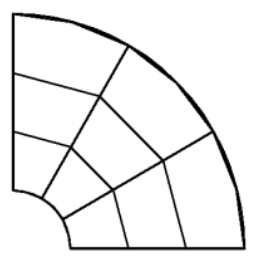

(b)

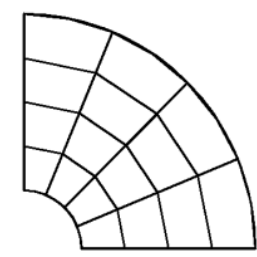

(c)

Fig. 4. Three levels of me sh for hollow cylinder: (a) $2 \times 2 \times 1$ mesh with total of 4 VPHEs (b) $3 \times 3 \times 1$ mesh with total of 9 VPHEs (c) $4 \times 4 \times 1$ mesh with total of 16 VPHEs.

The second case considered (Case 2) is determination of steady state temperature distribution within a square plate that is subjected to different temperature loads at the four sides of the plate. Geometry of the problem domain is shown in Fig. 5.

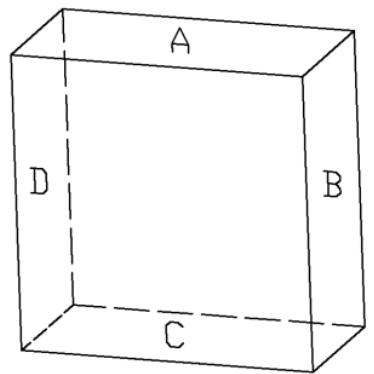

Fig. 5. Problem geometry of a square plate.

Surface A is subjected to fixed temperature of $100^{\circ} \mathrm{C}$ while surfaces $\mathrm{B}, \mathrm{C}$ and D are subjected to fixed temperature of $0^{\circ} \mathrm{C}$. Temperature distribution within the plate is calculated numerically by utilizing VPHEs in three different meshes that consist of 2, 4 and 21 elements, respectively, as shown in Fig. 6.

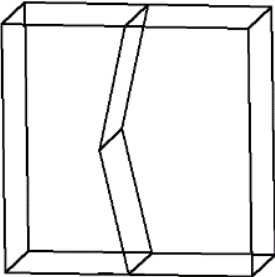

(a)

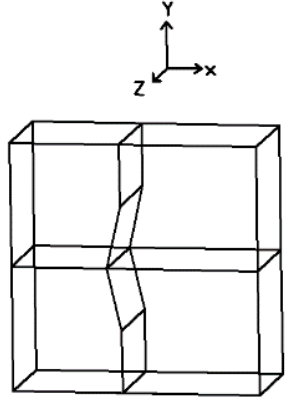

(b)

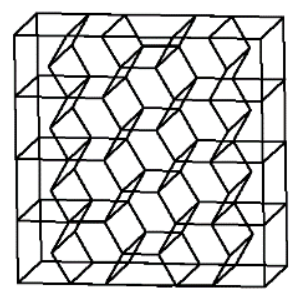

(c)

Fig. 6. Discretization of the problem domain by utilizing three different meshes: (a) 2 VPHEs with total of 32 global nodes (b) 4 VPHEs with total of 56 global nodes (c) 21 VPHEs with total of 240 global nodes.

Material properties for Cases 1 and 2 are similar to those utilized in patch test as described in section II. The third example (case 3) involves simulation of carbon nanotubes (CNT) [5]. Two configurations of CNT that are armchair and zigzag are modelled by utilizing VPHEs and their Young's modulus of elasticity are determined numerically. First, a cell of CNT is represented by a VPHE by positioning the nodes according to the carbon atoms in the unit cell, as shown in Fig. 7. 


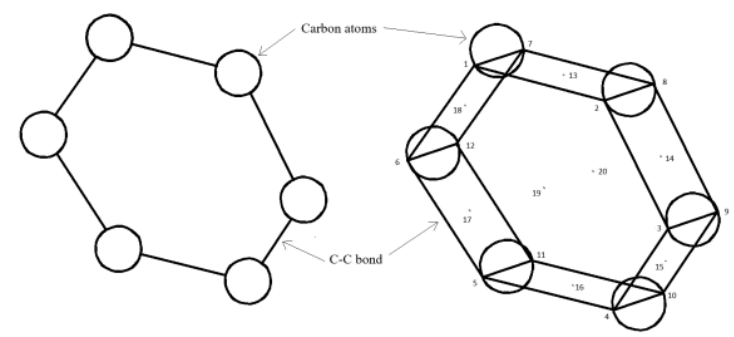

Fig. 7. Representation of a cell of CNT with VPHE: (a) a cell of CNT consisting of 6 carbon atoms, forming a hexagon (b) Equivalent VPHE element which represents the cell of CNT in 3-D.

The cell is then arranged in repeating pattern according to specific configuration to form the CNT with certain diameter, length and thickness. Fig. 8 shows an example of CNT with zigzag configuration of $(15,0)$ formed by the VPHEs. A single VPHE (which is repeated to form the entire CNT structure) is also shown in Fig. 8.

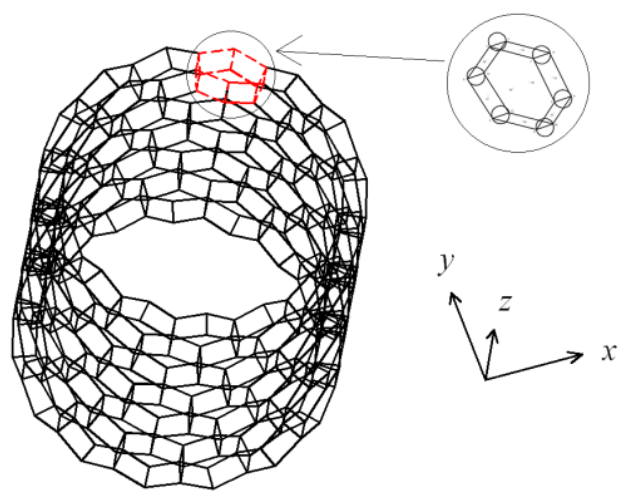

Fig. 8. A 3-D nano-scale continuum modelling of SWCNT by utilizing VPHE elements.

Young's modulus and Poisson's ratio for a single layer graphene are taken as $235.88 \mathrm{~N} / \mathrm{m}$ and 0.4136 , respectively [6]. Since CNTs are produced by rolling a single graphene sheet, therefore values of Young's modulus and Poisson's ratio can be assigned to the cells of CNT (see Fig. 7). For the modelling of CNT through VPHEs, the 2-D value of the Young's modulus is converted to 3-D, by dividing with thickness of the CNT [7], which then gives $E_{V P H E}=0.69 \mathrm{TPa}$. The length of C-C bond is taken as $0.142 \mathrm{~nm}$. Young's modulus of the entire CNT structures that are obtained from the simulations are then compared with other published data in the literature. Simulation results are shown in next section.

\section{Simulation Results}

Simulation results for the three cases considered in section III are presented here.

\subsection{Results for Case 1}

Steady state temperatures within the hollow cylinder are determined numerically by running simulations in Mathematica. The results are later compared with the analytical solution [8]:

$$
T(r)=\frac{\left(T_{2}-T_{1}\right)}{\ln \left(\frac{R_{2}}{R_{1}}\right)}\left(\ln (r)-\ln \left(R_{1}\right)\right)+T_{1}
$$

where $r=$ arbitrary radial distance, $T_{1}=$ temperature at the inner surface, $T_{2}=$ temperature at the outer surface, $R_{1}=$ radius of the inner surface and $R_{2}=$ radius of the outer surface. Percentage error is found 
using:

$$
\% \text { error }=\left(\frac{\mid \text { Exact value }- \text { Calculated value }}{\text { Exact value }}\right) \times 100 \%
$$

Simulation results are shown in Fig. 9. It can be seen that the VPHEs are able to yield correct solutions. Smaller percentage of error is obtained when higher numbers of elements are utilized.

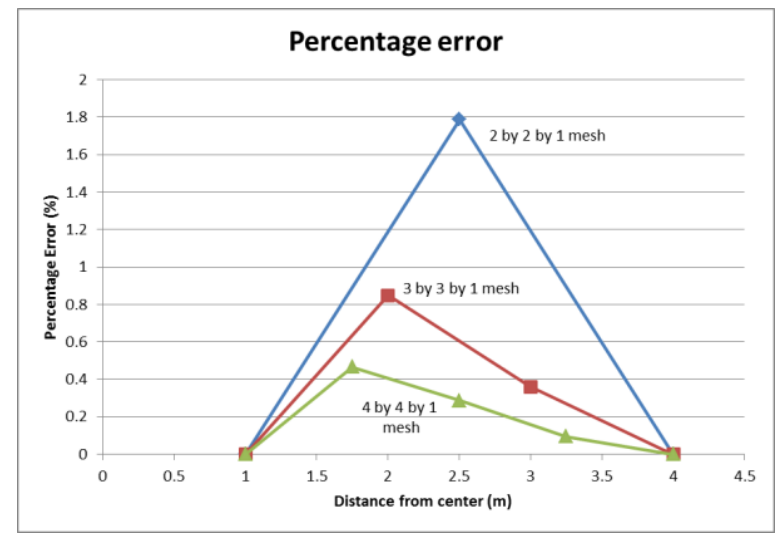

Fig. 9. Convergence of the solution for the temperatures.

\subsection{Results for Case 2}

Steady state temperatures within the square plate are determined numerically by running simulations in Mathematica. The results are later compared with the analytical solution which is given in [9]:

$$
\theta(x, y)=\frac{2}{\pi} \sum_{n=1}^{\infty} \frac{(-1)^{n+1}+1}{n} \sin \frac{n \pi x}{L} \frac{\sinh (n \pi y / L)}{\sinh (n \pi W / L)}
$$

where $L$ and $W$ represent length and width of the plate, respectively. The exact solution is obtained by taking $n$ as 10,000. Percentage error is found using (5). Simulation results are shown in Fig. 10.

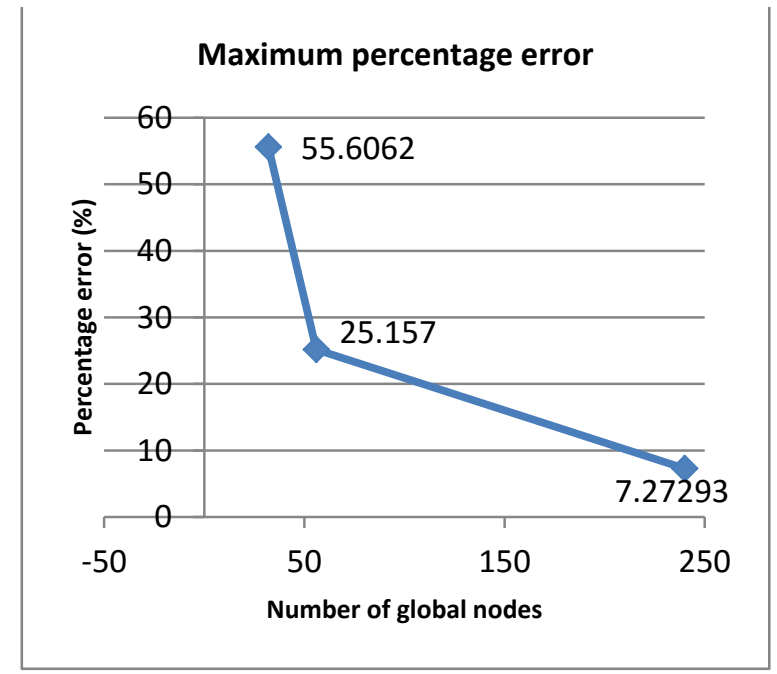

Fig. 10. Convergence of the solutions with increasing number of global nodes.

The maximum error of $7.27 \%$ is caused by the temperature difference between $2.17441^{\circ} \mathrm{C}$ and $2.01627^{\circ} \mathrm{C}$. This error can be eliminated when the temperatures are rounded to the nearest digit. 


\subsection{Results for Case 3}

Young's modulus of various CNT models $\left(E_{C N T}\right)$ with different parameters are determined through tensile test and the results are compared with other published data. The tensile test is simulated by fixing one end of the CNT and the other end is subjected to a predetermined displacement of $0.0023 \mathrm{~nm}$ (within the elastic region). Net reaction force is calculated by summing all the forces obtained at the fixed end of the CNT. Young's modulus for entire CNT model, $E_{C N T}$ is then calculated by utilizing following formulas [10]-[14]:

$$
\begin{aligned}
& K_{C N T}=\sum F / \delta \\
& E_{C N T}=\left(K_{C N T} L\right) / \pi D t
\end{aligned}
$$

where $\sum F$ represents net reaction force at the fixed end of CNT and $\delta$ represents prescribed displacement at the free end of CNT. $K_{C N T}, L, D$ and $t$ represent stiffness, length, diameter and thickness of the CNT. Simulations are carried out in Mathematica environment and the results are shown in Table 2.

\begin{tabular}{|c|c|c|c|c|c|}
\hline Method & References & Year & Remarks & $\begin{array}{l}\text { Wall thickness } \\
(\mathrm{nm})\end{array}$ & Young's modulus (TPa) \\
\hline Experimental & [15] & 1997 & - & - & 1.28 \\
\hline \multirow[t]{6}{*}{${ }^{\mathrm{a} A M}$} & [16] & 1998 & Tight binding ( $\mathrm{d} M D$ ) & 0.34 & 1.24 \\
\hline & [17] & 1999 & Tight binding ( $\left.{ }^{\mathrm{d}} \mathrm{MD}\right)$ & 0.34 & 1.24 \\
\hline & [18] & 2003 & $\begin{array}{l}\text { Energy approach } \\
\left({ }^{\mathrm{d}} \mathrm{MD}\right)\end{array}$ & 0.34 & 1.238 \\
\hline & [18] & 2003 & Force approach ( $\left.{ }^{\mathrm{d}} \mathrm{MD}\right)$ & 0.34 & 1.35 \\
\hline & [19] & 2006 & Ab initio & 0.34 & $1.23-1.36$ \\
\hline & [20] & 2009 & $\begin{array}{l}\text { Tight binding ( } \mathrm{d} M D \text { ) } \\
\text { and ab initio }\end{array}$ & - & $\begin{array}{l}0.95-1.33 \text { (MD) and } 1.02 \\
\text { (ab initio) }\end{array}$ \\
\hline${ }^{\mathrm{b}} \mathrm{CM}$ & [21] & 2008 & FEM spring elements & 0.34 & 1.2478 \\
\hline \multirow[t]{4}{*}{ cNCM } & [14] & 2012 & $\begin{array}{l}\text { FEM nonlinear spring } \\
\text { elements }\end{array}$ & 0.34 & 1.325 \\
\hline & [22-29] & $\begin{array}{l}2003- \\
2015\end{array}$ & FEM beam element & 0.34 & $0.81-1.4$ \\
\hline & {$[30]$} & 2016 & FEM beam element & $\begin{array}{l}\text { Other than } \\
0.34\end{array}$ & $1.27 \pm 0.02$ \\
\hline & Current work & $\begin{array}{l}2016- \\
2017\end{array}$ & Polyhedral FEM & 0.34 & $\begin{array}{l}\text { average } 1.2 \text { (Armchair) } \\
\text { average } 1.3 \text { (Zigzag) }\end{array}$ \\
\hline${ }^{\mathrm{a} A M}$ and ${ }^{\mathrm{c} N C M}$ & {$[22]$} & 2009 & $\begin{array}{l}\text { dMD and FEM (using } \\
\text { spring and beam } \\
\text { elements) }\end{array}$ & 0.34 & $1.2,1.4$ \\
\hline
\end{tabular}

Table 2. Comparative Study on Young's Modulus

Results from the current work are in good agreement with other published works. For example, the average Young's modulus of 1.2 TPa which is obtained for armchair configuration from this work is close to the results presented in [15]-[18], [21], [22]. Average value of $1.3 \mathrm{TPa}$ which is obtained for zigzag configuration in current work is similar to the results presented in [14] and [18].

\section{Conclusion}

New polyhedral elements known as VPHEs have been successfully developed and validated in this work. The polyhedral elements which are proposed in this paper can be utilized to analyse problems which arise in solid mechanics and heat transfer phenomena, as demonstrated through several examples (hollow cylinder, square plate and carbon nanotubes). One of the advantages of the VPHEs is that the nodes can be placed randomly in order to form arbitrary shape. This enables better representation of irregular boundaries as shown in the case of curved boundary in case 1, Fig. 4. Apart from that, the VPHEs can be 
utilized to generate better plot for temperature distribution within a square plate due to higher number of nodes for each element, as shown in case 2, Fig. 10. Finally, VPHEs are shown to be ideal in modelling CNTs with results that match other published works.

\section{Acknowledgment}

The first author would like to thank Research Management Centre (RMC) of Multimedia University, Malaysia, for providing financial support through Mini Funds with Grant nos. MMUI/130070 and MMUI/160047, which enabled purchase of required software and equipment for this work.

\section{References}

[1] Logah, P., \& Thet, T. M. (2011). Finite elements for engineering analysis: A brief review. International Proceedings of Computer Science and Information Technology, Vol. 10, (pp. 60-68).

[2] Logah, P. (2016). A novel virtual node hexahedral element with exact integration and octree meshing. Mathematical Problems in Engineering, 1-19.

[3] Sukumar, N., \& Malsch, E. A. (2006). Recent advances in the construction of polygonal finite element interpolants. Archives of Computational Methods in Engineering, 13, 129-163.

[4] Manzini, G., Russo, A., \& Sukumar, N. (2014). New perspectives on polygonal and polyhedral finite element methods. Mathematical Models and Methods in Applied Sciences, 24, 1665-1699.

[5] Logah, P., Lim, T. L., \& Tso, C. P. (2016). Nano-scale continuum modelling of carbon nanotubes by polyhedral finite elements. Journal of Nanomaterials, 1-9.

[6] Zhou, J., \& Huang, R. (2008). Internal lattice relaxation of single-layer graphene under in-plane deformation. Journal of the Mechanics and Physics of Solids, 56, 1609-1623.

[7] Kalosakas, G., Lathiotakis, N. N., Galiotis, C., \& Papagelis, K. (2013). In-plane force fields and elastic properties of graphene. Journal of Applied Physics, 113, 134307.

[8] Lienhard, J. H. (1981). A Heat Transfer Textbook. Englewood Cliffs, NJ: Prentice-Hall.

[9] Frank, P. I., \& David, P. D. (2002). Fundamentals of Heat and Mass Transfer. US: John Wiley.

[10] Tserpes, K., \& Papanikos, P. (2005). Finite element modelling of single-walled carbon nanotubes. Composites Part B: Engineering, 36(5), 468-477.

[11] Zuberi, M. J., \& Esat, V. (2015). Evaluating the effects of size and chirality on the mechanical properties of single-walled carbon nanotubes through equivalent-continuum modelling. Proceedings of the Institution of Mechanical Engineers, Part L: Journal of Materials: Design and Applications: Vol. 230, (pp. 913-926).

[12] Lu, X., \& Hu, Z. (2012). Mechanical property evaluation of single-walled carbon nanotubes by finite element modelling. Composites Part B: Engineering, 43(4), 1902-1913.

[13] Mohammadpo, E., Awang, M., \& Abdullah, M. (2011). Predicting the young's modulus of single-walled carbon nanotubes using finite element modelling. Journal of Applied Sciences, 11, 1653-1657.

[14] Rafiee, R., \& Heidarhaei, M. (2012), Investigation of chirality and diameter effects on the young's modulus of carbon nanotubes using non-linear potentials. Composite Structures, 94, 2460-2464.

[15] Wong, E. W., Sheehan, P. E., \& Lieber, C. M. (1997). Nanobeam mechanics: elasticity, strength, and toughness of nanorods and nanotubes. Science, 277, 1971-1975.

[16] Hernández, E., Goze, C., Bernier, P., \& Rubio, A. (1998). Elastic properties of C and B x C y N z composite nanotubes. Physical Review Letters, 80, 4502-4505.

[17] Goze, C., Vaccarini, L., Henrard, L., Bernier, P., Hemandez, E., \& Rubio, A. (1999). Elastic and mechanical properties of carbon nanotubes. Synthetic Metals, 103, 2500-2501.

[18] Jin, Y., \& Yuan, F. G. (2003). Simulation of elastic properties of single-walled carbon nanotubes. 
Composites Science and Technology, 63, 1507-1515.

[19] Peng, Y., Zhang, L., Jin, Q., Li, B., \& Ding, D. (2006). Ab initio studies of elastic properties and electronic structures of C and BN nanotubes. Physica E: Low-dimensional Systems and Nanostructures, 33, 155-159.

[20] Cai, J., Wang, Y., \& Wang, C. (2009). Effect of ending surface on energy and young's modulus of single-walled carbon nanotubes studied using linear scaling quantum mechanical method. Physica B: Condensed Matter, 404, 3930-3934.

[21] Giannopoulos, G., Kakavas, P., \& Anifantis, N. (2008). Evaluation of the effective mechanical properties of single-walled carbon nanotubes using a spring based finite element approach. Computational Materials Science, 41, 561-569.

[22] Cheng, H., Liu, Y., Hsu, Y., \& Chen, W. (2009). Atomistic-continuum modelling for mechanical properties of single-walled carbon nanotubes. International Journal of Solids and Structures, 46, 1695-1704.

[23] Li, C., \& Chou, T. W. (2003). A structural mechanics approach for the analysis of carbon nanotubes. International Journal of Solids and Structures, 40, 2487-2499.

[24] Ávila, A. F., \& Lacerda, G. S. (2008). Molecular mechanics applied to single-walled carbon nanotubes. Materials Research, 11, 325-333.

[25] Wernik, J. M., \& Meguid, S. A. (2009). Atomistic-based continuum modelling of the nonlinear behavior of carbon nanotubes. Acta Mechanica, 212, 167-179.

[26] Shokrieh, M. M., \& Rafiee, R. (2010). Prediction of young's modulus of graphene sheets and carbon nanotubes using nanoscale continuum mechanics approach. Materials \& Design, 31, 790-795.

[27] Sakharova, N., Pereira, A., Antunes, J., Brett, C., \& Fernandes, J. (2015). Mechanical characterization of single-walled carbon nanotubes: Numerical simulation study. Composites Part B: Engineering, 75, 73-85.

[28] Mohammadpo, E., Awang, M., \& Abdullah, M. (2011). Predicting the young's modulus of single-walled carbon nanotubes using finite element modelling. Journal of Applied Sciences, 11, 1653-1657.

[29] Rahmandoust, M., \& Öchsner, A., (2012). On finite element modelling of single-and multi-walled carbon nanotubes. Journal of Nanoscience and Nanotechnology, 12, 8129-8136.

[30] Al-Kharusi, M. S., Alzebdeh, K., \& Pervez, T. (2016). An atomistic-based continuum modelling for evaluation of effective elastic properties of single-walled carbon nanotubes. Journal of Nanomaterials, 2016, 1-13.

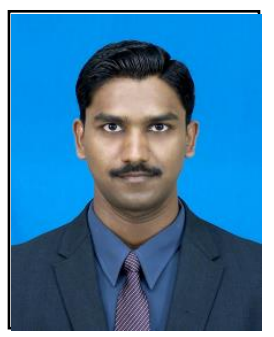

Logah Perumal received his bachelor degree in mechanical engineering and master in mechanical engineering from Universiti Tenaga Nasional, Malaysia. Currently he is working as a lecturer with Faculty of Engineering and Technology at Multimedia University, Malaysia. His research interests include numerical methods, finite element method, computation and fuzzy logic. He has published research articles at national and international journals, conference proceedings, and contributed to chapter of a book.

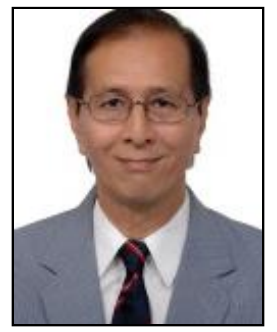

C. P. Tso obtained his bachelor's degree from Loughborough University, master's degree from Massachusetts Institute of Technology and Doctorate degree from University of California, Berkeley in 1979.

He is presently a professor in the Faculty of Engineering and Technology, Multimedia University, Malaysia. He has written extensively in technical papers and conference proceedings. His current interest is in heat and fluid flows. 
Prof. Tso is a fellow member of The Institution of Mechanical Engineers and The Institute of Physics, United Kingdom. He is also fellow member of The Institution of Engineers Malaysia and The Institution of Engineers Singapore. He is a chartered engineer and a professional engineer.

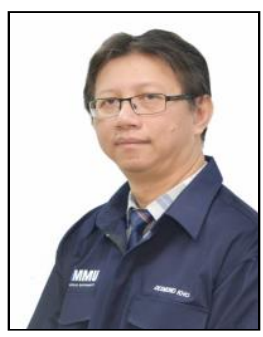

Lim Thong Leng obtained his first degree in physics from National University of Malaysia. He was awarded with Ph.D. in 2009. He is interested in computational material science, particularly in the field of semiconducting and thermoelectric material, nanomaterial and its characterization, DFTB and optimization. 